\title{
Hot and Cold: A Concurrent Warm and Cold Autoimmune Hemolytic Anemia in B-cell Prolymphocytic Leukemia
}

\author{
Jonathan Feld ${ }^{a}$ Jon Arnason $^{b}$ Kerry O'Brien ${ }^{b}$ Myrna Nahas ${ }^{b}$ \\ a Division of Hematology/Medical Oncology, Tisch Cancer Institute, Mount Sinai Hospital, Icahn School of \\ Medicine, New York, NY, USA; ${ }^{b}$ Beth Israel Deaconess Medical Center, Harvard Medical School, Boston, MA, USA
}

Dear Editor,

B-cell prolymphocytic leukemia (B$\mathrm{PLL})$ is a rare hematological malignancy with an estimated 150 cases per year in the United States [1]. Cold autoimmune hemolytic anemia (AIHA) is a similarly uncommon condition with a yearly incidence of 1 case per million [2].

An 84-year-old male with a 16-year history of p53-deleted chronic lymphocytic leukemia (CLL) and stage IV esophageal adenocarcinoma presented with worsening fatigue. His treatment for CLL with idelalisib had recently been held following esophagectomy. Admission laboratory results were significant for a hemoglobin ( $\mathrm{Hb}$ ) level of $8.5 \mathrm{~g} / \mathrm{dL}$, a mean corpuscular volume of $125 \mathrm{fL}, 7.1 \%$ reticulocytes, white blood cells (WBC) of $86.5 \mathrm{~K} / \mu \mathrm{L}$ (89\% lymphocytes), indirect bilirubin $2.1 \mathrm{mg} / \mathrm{dL}$, lactate dehydrogenase $478 \mathrm{IU} / \mathrm{L}$, and haptoglobin $<10 \mathrm{mg} /$ $\mathrm{dL}$. The peripheral blood smear showed spherocytes, and the direct antiglobulin test (DAT) was $2+$ for IgG and $3+$ for C3. These laboratory results signified acute hemolysis in the context of a marked in- crease in his CLL burden. He was promptly treated with methylprednisolone 100 $\mathrm{mg}$ daily, rituximab $375 \mathrm{mg} / \mathrm{m}^{2}$, and idelalisib, which was restarted at $175 \mathrm{mg}$ twice a day.

Peripheral blood flow cytometry demonstrated $91.3 \%$ lymphocytes, $95 \%$ of which were CD19+/CD20+, kappa-restricted B cells. Flow detected a small subset of dim CD5+ cells, whereas previous flow showed co-expression of CD5 with CD19/CD20. The corresponding peripheral blood smear had 54\% prolymphocytes with prominent nucleoli and minimal cytoplasm. Cytogenetics revealed trisomy 12,13q14, and TP53 deletions. Blood bank testing identified warm autoantibodies as well as cold autoantibodies, due to the DAT being positive for complement (C3) as well as IgG, agglutination of the patient's blood at room temperature, and the observation that prewarming the blood reduced unexpected reactivity in the patient's front typing $(\mathrm{ABO})$ tests. The patient's eluate showed panagglutination consistent with the warm autoantibody.
During his hospital admission, the patient's $\mathrm{Hb}$ eventually improved to $8.0 \mathrm{~g} /$ $\mathrm{dL}$ (nadir was $6.4 \mathrm{~g} / \mathrm{dL}$ ), and WBCs decreased to $72.6 \mathrm{~K} / \mu \mathrm{L}$. The DAT remained $3+$ for IgG, although the strength of the reactivity for $\mathrm{C} 3$ decreased to $1+$ within 1 week of starting treatment (Fig. 1). He was discharged on idelalisib, prednisone, and weekly rituximab. Within 1 month of initiating treatment, his $\mathrm{Hb}$ reached $10 \mathrm{~g} /$ $\mathrm{dL}$, and by 2 months, the haptoglobin level normalized and leukocytosis resolved. The patient's last dose of rituximab was 1 month after his initial presentation, and prednisone was tapered down to $30 \mathrm{mg}$ daily by month 2 . The use of idelalisib appeared to effectively treat his B-PLL; 2 months following initiation of therapy, his WBC count was $9.5 \mathrm{~K} / \mu \mathrm{L}$ with a normal differential. Unfortunately, the patient expired 3 months after transformation due to comorbidities associated with his esophageal adenocarcinoma.

While CLL is a common hematological malignancy, transformation to B-PLL is rare [3]. Autoimmune hemolysis occurs in $10-25 \%$ of all CLL [4] and in up to
Jonathan Feld, MD

Division of Hematology/Medical Oncology, Tisch Cancer Institute Mount Sinai Hospital, 1 Gustave L. Levy Place, Box 1079 New York, NY 10029 (USA)

E-Mail jonathan.feld@ mountsinai.org 


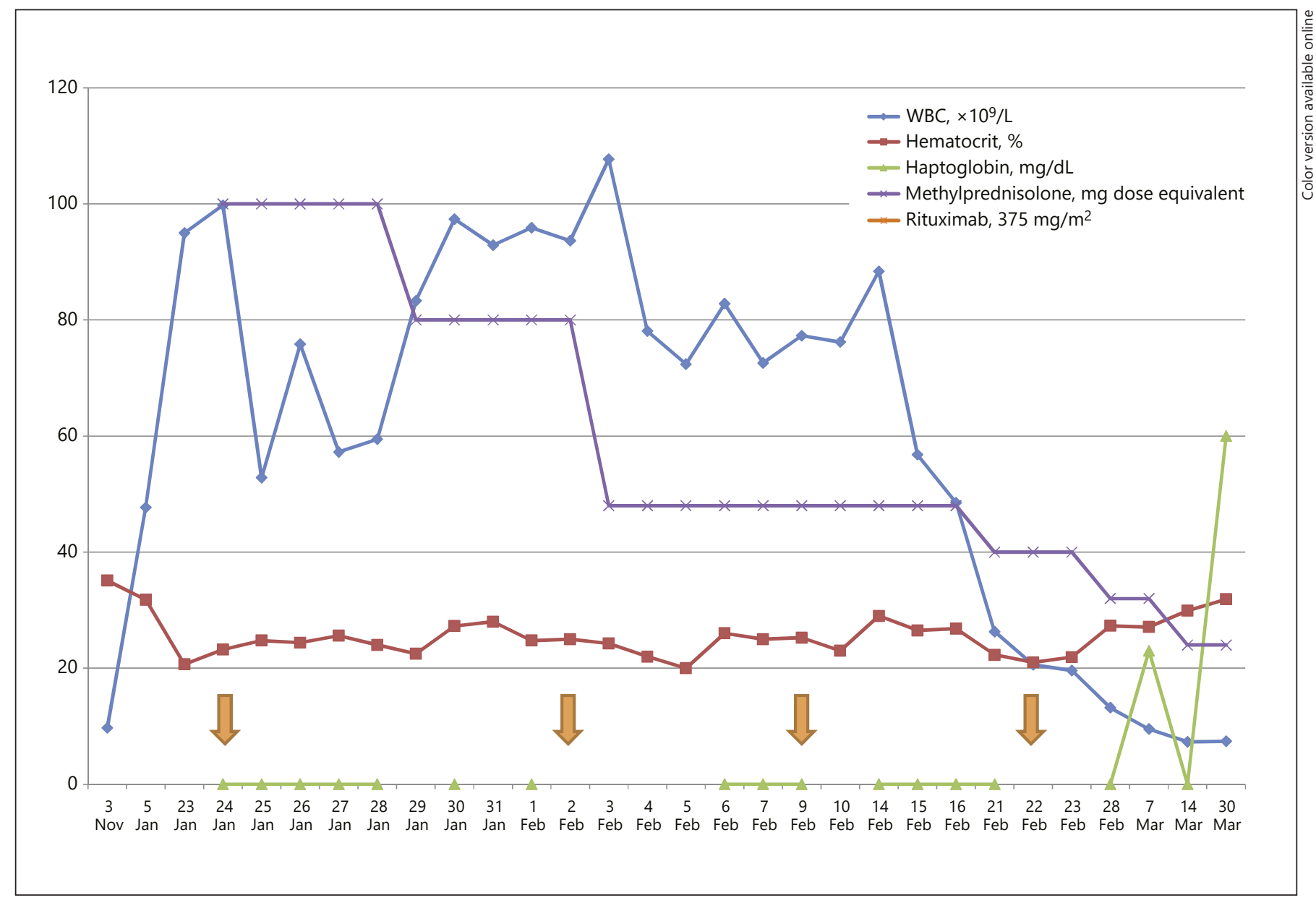

Fig. 1. Trend of WBC count, hematocrit, and haptoglobin in response to intermittent rituximab treatments and a steroid taper (in methylprednisolone dose equivalents).

$10 \%$ of the B-PLL cases [5]. Interestingly, previous studies have shown a correlation between cold agglutinin and cytogenetic abnormalities including trisomy $3, \mathrm{t}(8$; 22 ), and, as in our case, trisomy 12 [6]. In both B-PLL and CLL, warm AIHA is much more frequently reported than cold AIHA [7]. In fact, there has been only 1 prior reported case of cold agglutinin associated with B-PLL. A combination of rituximab, fludarabine, and cyclophosphamide improved the leukocytosis, normalized the $\mathrm{Hb}$, and resolved the hemolysis [8]. However, that case was not associated with concurrent transformation of CLL to B-PLL, nor was there a concomitant warm AIHA. In our literature review, we were unable to find a previously reported case of a mixed cold and warm AIHA occurring in B-PLL. Here, we re-

A Concurrent Warm and Cold AIHA in

B-cell Prolymphocytic Leukemia port the novel and effective use of a combination of rituximab, prednisone, and idelalisib in reversing a rare cold and warm AIHA in the context of transformed CLL to B-PLL.

The degree to which idelalisib contributed to the resolution of hemolysis remains unknown, though its use does not appear to have had significant adverse effects. Notably, recent recommendations suggest avoiding idelalisib in the context of CLL-associated autoimmune cytopenias, due to its potential immunomodulating effect [9], and there are no published prior cases of using idelalisib to control AIHA. AIHA was not a reported toxicity in a recent case series of 8 patients (7 with TP-53 deletions) with B-PLL treated with idelalisib and rituximab [10]. Our case reflects an innovative and effi- cacious therapeutic regimen, consisting of prednisone, rituximab, and idelalisib, when facing an uncommon mixed AIHA associated with an aggressive TP-53-deleted B-PLL.

\section{Disclosure Statement}

The authors have no conflicts of interest to declare.

\section{Funding Sources}

The authors did not receive any funding. 


\section{References}

1 Yamamoto JF, Goodman MT. Patterns of leukemia incidence in the United States by subtype and demographic characteristics, 1997 2002. Cancer Causes Control. 2008 May; 19(4):379-90.

2 Berentsen S, Ulvestad E, Langholm R, Beiske K, Hjorth-Hansen H, Ghanima W, et al. Primary chronic cold agglutinin disease: a population based clinical study of 86 patients. Haematologica. 2006 Apr;91(4):460-6.

3 Rana C, Sharma S, Agarwal M. Prolymphocytic and Richter's Transformation in Peripheral Blood: A Case Report and Review of Literature. J Hematol (Brossard). 2014;3(3):868.
4 Duek A, Shvidel L, Braester A, Berrebi A. Clinical and immunologic aspects of $B$ chronic lymphocytic leukemia associated with autoimmune disorders. Isr Med Assoc J. 2006 Dec;8(12):828-31.

5 Roberts JC, Roberts GH. B-Prolymphocytic leukemia: a case study. Clin Lab Sci. 2001 Fall; 14(4):233-7.

6 Chng WJ, Chen J, Lim S, Chong SM, Kueh YK, Lee SH. Translocation $(8 ; 22)$ in cold agglutinin disease associated with B-cell lymphoma. Cancer Genet Cytogenet. 2004 Jul; 152(1):66-9.

7 Mauro FR, Foa R, Cerretti R, Giannarelli D, Coluzzi S, Mandelli F, et al. Autoimmune hemolytic anemia in chronic lymphocytic leukemia: clinical, therapeutic, and prognostic features. Blood. 2000 May;95(9):2786-92.
8 Yiu RC, Tien SL. Cold agglutinin disease with prolymphocytic leukemia. Blood. 2012 May; 119(22):5069.

9 Tsang M, Parikh SA. A Concise Review of Autoimmune Cytopenias in Chronic Lymphocytic Leukemia. Curr Hematol Malig Rep. $2017 \mathrm{Feb} ; 12(1): 29-38$.

10 Eyre TA, Fox CP, Boden A, Bloor A, Dungawalla $\mathrm{M}$, Shankara $\mathrm{P}$, et al. Idelalisib-rituximab induces durable remissions in TP53 disrupted B-PLL but results in significant toxicity: updated results of the UK-wide compassionate use programme. Br J Haematol. DOI:10.1111/bjh.15151. 